\title{
Local Entrainment of Alpha Oscillations by Visual Stimuli Causes Cyclic Modulation of Perception
}

\author{
Eelke Spaak, Floris P. de Lange, and Ole Jensen \\ Donders Institute for Brain, Cognition, and Behaviour, Centre for Cognitive Neuroimaging, Radboud University Nijmegen, 6525 EN Nijmegen, \\ The Netherlands
}

\begin{abstract}
Prestimulus oscillatory neural activity in the visual cortex has large consequences for perception and can be influenced by top-down control from higher-order brain regions. Making a causal claim about the mechanistic role of oscillatory activity requires that oscillations be directly manipulated independently of cognitive instructions. There are indications that a direct manipulation, or entrainment, of visual alpha activity is possible through visual stimulation. However, three important questions remain: (1) Can the entrained alpha activity be endogenously maintained in the absence of continuous stimulation?; (2) Does entrainment of alpha activity reflect a global or a local process?; and (3) Does the entrained alpha activity influence perception? To address these questions, we presented human subjects with rhythmic stimuli in one visual hemifield, and arhythmic stimuli in the other. After rhythmic entrainment, we found a periodic pattern in detection performance of near-threshold targets specific to the entrained hemifield. Using magnetoencephalograhy to measure ongoing brain activity, we observed strong alpha activity contralateral to the rhythmic stimulation outlasting the stimulation by several cycles. This entrained alpha activity was produced locally in early visual cortex, as revealed by source analysis. Importantly, stronger alpha entrainment predicted a stronger phasic modulation of detection performance in the entrained hemifield. These findings argue for a cortically focal entrainment of ongoing alpha oscillations by visual stimulation, with concomitant consequences for perception. Our results support the notion that oscillatory brain activity in the alpha band provides a causal mechanism for the temporal organization of visual perception.
\end{abstract}

Key words: alpha oscillations; entrainment; rhythmic stimulation; visual perception

\section{Introduction}

It has been well established that prestimulus neural activity correlates with visual perception. In particular, both the power and the phase of prestimulus occipital alpha oscillations $(8-12 \mathrm{~Hz})$ can determine whether a near-threshold target is detected or not (Hanslmayr et al., 2007; van Dijk et al., 2008; Busch et al., 2009; Mathewson et al., 2009; Thut et al., 2012). These oscillatory processes are likely under top-down control from higher-order brain regions (Capotosto et al., 2009, 2012). Typically when an effect of oscillatory activity on perception is shown, the analysis is correlative. However, it is desirable to go beyond such correlative analyses and ask: Is there a causal relationship between oscillatory neural activity and perception?

One potentially fruitful avenue of research is the manipulation of endogenous oscillations by external stimuli through entrainment. For instance, transcranial magnetic stimulation (TMS) at alpha frequencies has been shown to affect oscillatory

\footnotetext{
Received 0ct. 14, 2013; revised Jan. 14, 2014; accepted Jan. 25, 2014.

Author contributions: E.S., F.P.d.L., and 0.J. designed research; E.S. performed research; E.S. analyzed data; E.S., F.P.d.L., and 0.J. wrote the paper.

This work was supported by a Netherlands Organization for Scientific Research (NWO) Vici Grant\#453-09-002 (to 0.J. and E.S.), NWO-ALW Open Competition Grant No. 822-02-011 (to 0.J.), and a James S. MCDonnell Foundation Scholar Award (to F.d.L.).

The authors declare no competing financial interests.

Correspondence should be addressed to Ole Jensen at the above address. E-mail: ole.jensen@donders.ru.nl.

DOI:10.1523/JNEUROSCI.4385-13.2014

Copyright $\odot 2014$ the authors $\quad 0270-6474 / 14 / 333536-09 \$ 15.00 / 0$
}

activity as measured by electroencephalography (EEG; Thut et al., 2011) and perceptual performance (Romei et al., 2010). The entrainment of endogenous oscillations by visual stimulation has also been investigated. It has been shown that the human visual cortex shows the strongest resonance to $10 \mathrm{~Hz}$ visual flicker (Herrmann, 2001). Furthermore, a perceptual aftereffect in the form of a rhythmic modulation of hit rate after $10 \mathrm{~Hz}$ visual stimulation has been reported (de Graaf et al., 2013), as well as phase-locking of neural oscillatory activity during such stimulation (Mathewson et al., 2012). Finally, although there are indications in the literature that neural activity following rhythmic stimulation is similar to neural activity observed during stimulation (Halbleib et al., 2012), persistent poststimulus entrainment effects in terms of increased phase-locking or power modulation has not yet been demonstrated.

Summarizing, three important open questions remain concerning the entrainment of endogenous alpha oscillations using visual stimulation. First, can the entrained alpha activity be endogenously maintained in the absence of continuous stimulation? Second, does entrainment of alpha activity reflect a global or a local process? And third, does the entrained alpha activity influence perception? The present study addresses these three questions, using a paradigm involving bilateral visual flicker stimulation and difficult-to-detect visual targets, as well as continuous recording of neural activity using magnetoencephalography (MEG). These techniques enabled us to investigate the 
effect of visual flicker on both behavioral performance and brain oscillations simultaneously.

\section{Materials and Methods}

Participants. Twenty-two subjects ( 7 female; age range, $18-51$ years) with no history of neurological or psychiatric disorders (based on self-report) participated in this experiment. All subjects gave written informed consent according to the Declaration of Helsinki. The study was approved by the local ethics committee (CMO region Arnhem/Nijmegen). Data for two subjects had to be excluded because of excessive eye movements, and a further subject was excluded because detection performance was at chance level. This leaves a total of 19 subjects on which all analyses are based.

Experimental design. During the entire experiment, a fixation cross was presented against a gray background. During the intertrial interval (1.5 s), the fixation cross was dimmed, indicating to the participant that blinking or making saccades was allowed. At the beginning of each trial, a bright fixation cross was presented for $1 \mathrm{~s}$, instructing the participant to refrain from blinking or making saccades. After this baseline interval, a $1.5 \mathrm{~s}$ train of visual flashes was presented (Fig. 1A) in the form of white squares. Each flash lasted $17 \mathrm{~ms}$ (one frame refresh of our projector), the sides of each square measured $6^{\circ}$ (visual degrees), and the squares were placed at $3^{\circ}$ eccentricity. At each trial, either the left or the right visual field was presented with a $10 \mathrm{~Hz}$ periodic stimulus train (interflash interval, $83 \mathrm{~ms}$; total number of flashes per hemifield per trial, 16) while the other hemifield was presented with a jittered stimulus train. The timings of the jittered stimulus train were generated randomly, subject to the constraint that no two flashes could occur in subsequent frame refreshes (i.e., there always was at least one blank frame between two flashes). The number of flashes in both hemifields was kept the same, as was the timing of the first and last flashes. This controls for possible nonentrainmentspecific effects of the final entrainment flash, such as forward visual masking or a transient-evoked response in the MEG data. After a variable delay of 17-340 ms (in steps of $17 \mathrm{~ms}$, uniform probability across different possible delays), a circular aperture near-threshold sine-wave grating target (duration, $17 \mathrm{~ms}$; diameter, $1^{\circ}$ ) was presented in either of the two hemifields at the location of the flashing stimulus trains. The subjects' task was to identify, by a right-hand index or middle-finger button press, in which hemifield they detected this target (forced choice). After the left/right response, subjects had to indicate whether they were sure or unsure about their response. We do not report any results related to the confidence rating here, as this rating did not correlate with the alpha entrainment phenomenon under investigation.

To acquaint the subject with the task, each experimental session started with 40 practice trials with easy-to-detect targets. Next followed 60 trials in which we estimated the subjects' perceptual threshold to achieve an overall performance of $80 \%$ correct. To this end we used the Bayesian Quest procedure (Watson and Pelli, 1983) to adjust the contrast of the target such that the desired performance level was achieved. Next followed four blocks of 90 trials each. All analyses are based on the trials from these four blocks. The target grating contrast was kept constant throughout each block, but was updated between blocks (by updating the Quest distribution estimated after each trial) to accommodate effects of fatigue, habituation, or sensitization. In this way the performance of each subject was kept at the desired $80 \%$.

Experimental equipment. Stimuli were presented by back-projection onto a semitranslucent screen by an Eiki LC-XL100L projector. The projection measured $46 \mathrm{~cm}$ in width, and had a resolution of $1024 \times 768$ pixels. Subjects were seated in a magnetically shielded room, at a distance of $80 \mathrm{~cm}$ from the projection screen. Throughout the experiment, the MEG was recorded at a sampling rate of $1.2 \mathrm{kHz}$ using a 275-channel axial gradiometer CTF MEG system. In addition, we continually recorded subjects' gaze position using an SR Research Eyelink 1000 eyetracking device. Whenever the fixation cross was white, subjects could not blink or saccade further than $1.7^{\circ}$ from the fixation center. If they failed to maintain fixation, the trial was aborted and subjects were informed of their eye movement by a bright red cross on the screen. After the MEG experimental session, structural magnetic resonance imaging
(MRI) images were obtained from all subjects using a $1.5 \mathrm{~T}$ Siemens Magnetom Avanto system.

Behavioral analysis. Statistical significance of the sinusoidal fit to the hit rate data (Fig. 1C) was assessed by using a Wald test (Fox, 1997). This test controls for the increase in degrees of freedom of the complex model (line plus sinusoid) when compared with the simpler model (line only). In addition, we performed a permutation test: the amplitude of the sinusoidal fit to the observed hit rate data was compared with the amplitudes of sinusoidal fits to randomized data. Randomized data were generated by randomly permuting the hit rate time course 1000 times. Finally, to rule out the possibility that the periodicity was driven by a single subject, we performed a bootstrap procedure: we sampled randomly, with replacement, from our subjects, 5000 times to obtain a $95 \%$ confidence interval on the circular-to-linear correlation between delay and hit rate.

To quantify the rhythmic entrainment effect observed in the hit rate profile in a single quantity, we defined an "entrainment index" (EI). Trials could have the target either in-phase or anti-phase with the entraining stimuli. Also, the targets could be either ipsilateral [entrained (E)] or contralateral [nonentrained (NE)] to the rhythmic stimuli. The ratio between hit rate (HR) for anti-phase and in-phase entrained trials was defined as the variable of interest. We normalized this variable by the corresponding ratio in the nonentrained trials, to account for the nonphase-specific hit rate increase with delay. This yielded the following expression for the entrainment index:

$$
\mathrm{EI}=\frac{\mathrm{HR}_{E, \text { anti }} / \mathrm{HR}_{E, \text { in }}}{\mathrm{HR}_{N E, \text { anti }} / \mathrm{HR}_{N E, \text { in }}}
$$

The EI thereby reflects the amount to which the rhythmic stimulus train caused a rhythmic effect in the hit rate.

MEG data preprocessing. All MEG data were analyzed using Matlab R2012a, either using custom-written scripts or the FieldTrip toolbox (Oostenveld et al., 2011; http://www.ru.nl/neuroimaging/fieldtrip/). As judged by visual inspection, excessively noisy sensors were removed from the data (on average, 0.8 sensors were removed per session), as were trials containing strong muscle or movement artifacts (on average, 5.8 trials were removed per session). All trials were demeaned and a linear trend was fitted and removed. Data were downsampled to $300 \mathrm{~Hz}$ to speed up processing. An independent component analysis was performed to identify and remove activity caused by cardiac activity. For each session, two components reflecting the QRS complex were removed, and the decomposed data were backprojected to sensor space.

We computed an approximation of the MEG planar gradient, using FieldTrip's ft_megplanar function. Considering planar, rather than axial, gradient data facilitates the interpretation of MEG results, as planar gradient maxima are located above neural sources (Hari and Salmelin, 1997; Bastiaansen and Knösche, 2000). For the event-related fields, we combined the resulting horizontal and vertical planar gradients by singularvalue decomposition per channel, projecting the data along the strongest direction. For the spectral analyses, we computed metrics separately for the horizontal and vertical planar gradients, and combined the two by computing the sum.

Before the event-related averaging (Fig. 2), the data were bandpass filtered in the range of $2-30 \mathrm{~Hz}$ using a finite impulse response filter. The filter order was set to three cycles of the lower bound of the passband.

Whenever plots and results of sensor-space analyses (Figs. 2, 3, 5) refer to a particular selection of sensors (left/right, ipsilateral/contralateral), we always refer to the posterior sensors overlying the left and right hemispheres, as defined by the CTF MEG manufacturer.

Spectral analysis. To get a time-frequency representation (TFR) of power and intertrial coherence (ITC), we used a sliding time window fast Fourier transform (FFT) approach. Frequencies of interest ranged from 2 to $30 \mathrm{~Hz}$ in steps of $1 \mathrm{~Hz}$. The time window was always such that it fit exactly four cycles of the frequency of interest, and it slid over the time axis in steps of $50 \mathrm{~ms}$. Each instance of the sliding time window was multiplied by a Hanning taper and Fourier-transformed, thus yielding a time-resolved complex Fourier spectrum. Power values pow $w_{n}(f, t)$ for trial $n$, frequency $f$, and time point $t$ were computed by squaring the absolute value of the Fourier coefficients $c_{n}(f, t)$, i.e., $\operatorname{pow}_{n}(f, t)=\left|c_{n}(f, t)\right|^{2}$, 
A

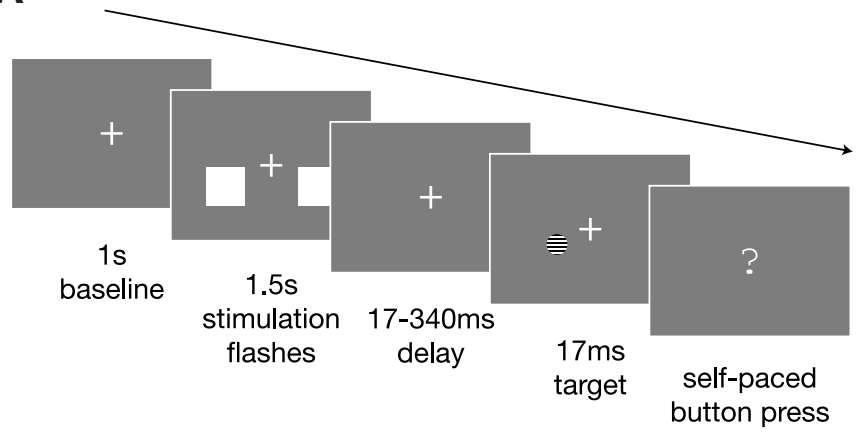

C

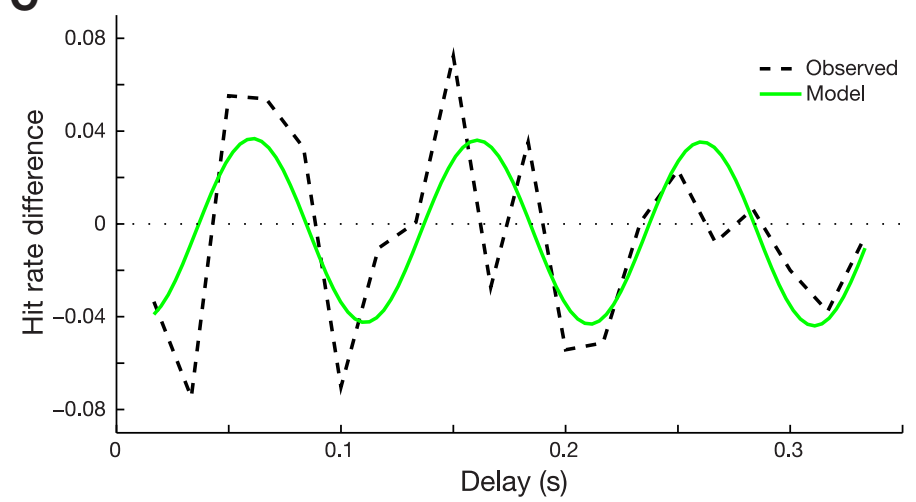

B

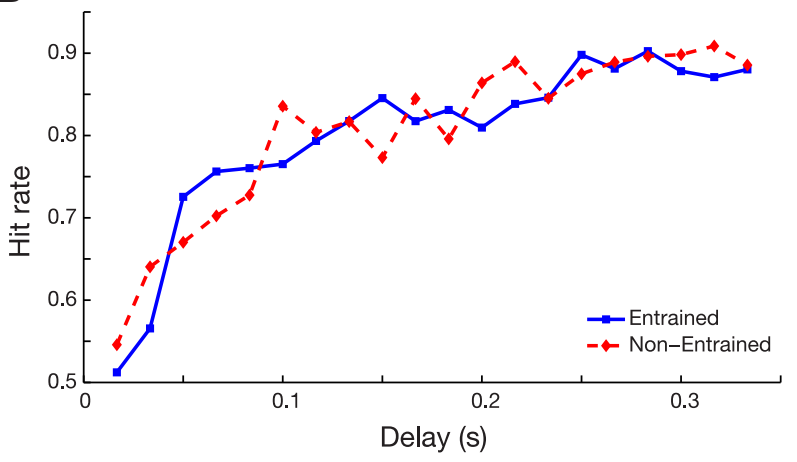

D

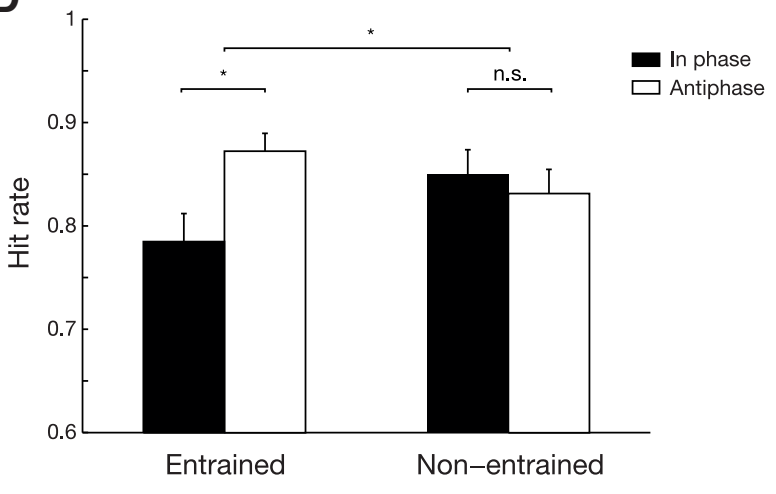

Figure 1. Experimental design and behavioral results. $\boldsymbol{A}$, Subjects were presented with 1.5 s stimulation trains composed of bilateral flashing white squares against a dark gray background. One of the squares flashed periodically at $10 \mathrm{~Hz}$. The other had a jittered interflash interval. The hemifield of random versus jittered stimuli was randomized over trials. After the offset of the stimulus trains, a near-threshold target appeared in either of the two hemifields after a brief random delay. Subjects were instructed to indicate whether they saw the target on the left or right (forced choice). $\boldsymbol{B}$, Hit rate as a function of the delay between entrainment stimuli and target. A clear improvement in performance was observed with increasing delay, both for the entrained and nonentrained trials. C, The difference in hit rate between entrained and nonentrained trials, as a function of delay. Dashed lines indicate grand average observed values. The green curve indicates the best fit $10 \mathrm{~Hz}$ sinusoid. $\boldsymbol{D}$, Hit rates computed for only in-phase $(100,200 \mathrm{~ms})$ or anti-phase $(150,250 \mathrm{~ms})$ delays, separately for the entrained and nonentrained trials. Repeated-measures ANOVA revealed a significant interaction between the two factors, and post hoc $t$ tests reveal that there is an effect of delay (i.e., phase) only when the target was presented at the entrained hemifield. Error bars reflect unbiased within-subjects corrected SEM (Cousineau, 2005; Morey, 2008). * $p<0.05$.

and were averaged over trials. Intertrial coherence was defined as the absolute value of the complex mean of the Fourier values, after normalizing them to unit length, as follows:

$$
\operatorname{ITC}(f, t)=\left|\frac{1}{N} \sum_{n=1}^{N} \frac{c_{n}(f, t)}{\left|c_{n}(f, t)\right|}\right|
$$

Source analysis. To obtain an estimate of the sources of the observed 10 $\mathrm{Hz}$ activity, we applied a beamforming spatial filtering technique known as dynamic imaging of coherent sources (DICS; Gross et al., 2001) to our $10 \mathrm{~Hz}$ power data. This step used the data recorded from the axial gradiometers. The DICS algorithm computes a spatial filter from the crossspectral density matrix (CSD) of the data and a lead field matrix. To compute the CSD, we used a multitaper FFT approach. Data were subselected between 0.9 and $1.5 \mathrm{~s}$ after entrainment stimuli onset, separately for data with entrainment in the right and left hemifield. Slepian tapers were applied to obtain a $\pm 2 \mathrm{~Hz}$ spectral smoothing, thus yielding an estimate of activity in the $8-12 \mathrm{~Hz}$ band. The CSD was estimated using the combined data of the left-entrained and right-entrained trials. To obtain the lead fields for each subject, we constructed a realistically shaped single-shell head model based on the individual anatomical MRI (Nolte, 2003), after spatially coregistering the MRI to sensor space MEG data by identifying fiducials in the nasion and the two ears. Each brain volume was divided into a grid of points spaced $8 \mathrm{~mm}$ apart, and warped to the template Montreal Neurological Institute (MNI) brain. The lead field was calculated for each grid point (Nolte, 2003). The estimated power in source space was averaged over trials. For visualization purposes, the grandaverage grid was interpolated onto the single-subject MNI template brain (Fig. 4).
The above approach yielded an estimate for the neural sources responsible for the alpha band power increase during the entraining stimuli. To get an estimate of the postentrainment active neural sources (Fig. 4B), we applied the filter, which was computed based on the $0.9-1.5 \mathrm{~s}$ time window, to the CSD estimated during the time window 1.5-2 s, i.e., after the entraining stimuli offset.

Cluster-based permutation statistics. To statistically quantify the timefrequency results of power and ITC, and the source analysis results, we performed cluster-based permutation tests (Maris and Oostenveld, 2007) across subjects. Specifically, for each voxel (either time/frequency/ channel voxels for sensor space TFR analyses or $x / y / z$ voxels for source space analysis) we computed the normalized difference between conditions: $(\mathrm{A}-\mathrm{B}) /(\mathrm{A}+\mathrm{B})$. This metric was computed both for the observed data and for 1000 (in the case of sensor space analysis) or 1500 (in the case of source space analysis) permutations of the conditions. Based on the per-voxel permutation distribution of descriptives thus obtained, we thresholded the observed values with the 95th percentile of this distribution to obtain cluster candidates. For each permutation, the cluster candidate with the highest sum of voxel-level descriptives was added to the permutation distribution of cluster statistics. The sum of descriptives for each observed cluster candidate was compared with this permutation distribution to assess significance for each cluster. For the source-level analysis, we used the maximum value of voxel-level descriptives per cluster (rather than the sum of all descriptives) as the cluster statistic to test, since spatial extent of clusters in beamforming typically is not closely related to the reliability of the source reconstruction (i.e., beamforming tries to make sources as small as possible; in the ideal case neighboring voxels are independent). For the sensor-level analysis, using the sum of 


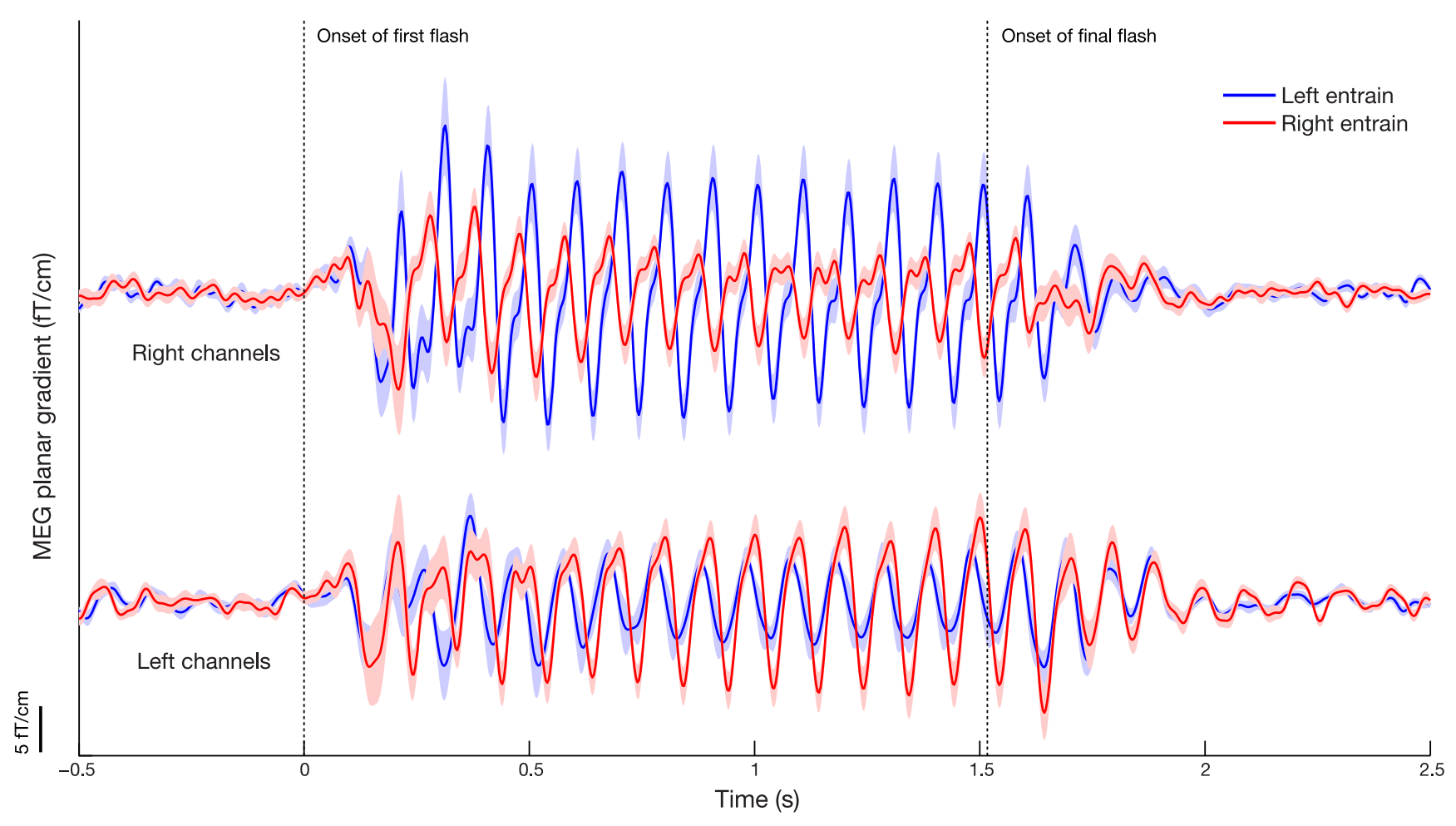

Figure 2. ERFs during rhythmic stimulation. The MEG planar gradient shown separately for the sensors overlying the left (bottom) and right (top) occipitoposterior hemispheres (as defined by the (TF MEG manufacturer). Red and blue curves correspond to rhythmic stimulation in the right and left visual fields, respectively. A periodic pattern is clearly visible as a consequence of the stimulation. Amplitude was higher over the contralateral sensors. Importantly, the rhythmicity in the ERFs persisted for several cycles after the end of the rhythmic stimulation (second vertical dotted line). The MEG planar gradient in the direction of most power was used, as computed by singular value decomposition. Error bounds reflect unbiased within-subjects corrected SEM (Cousineau, 2005; Morey, 2008).

descriptives makes more sense, as cluster extent is informative as to the reliability of the finding (i.e., neighboring time/frequency/channel points are likely to be highly correlated).

\section{Results}

Subjects were presented with trains of 16 flashes that were temporally spaced over a $1.5 \mathrm{~s}$ period against a gray background (Fig. $1 A)$. Each flash lasted $17 \mathrm{~ms}$. Either the left or the right visual field was stimulated by $10 \mathrm{~Hz}$ periodic flashing, while the other hemifield was stimulated by a jittered flash train. After a variable delay (17-340 ms), a near-threshold target (duration, $17 \mathrm{~ms}$ ) was presented in one of the two hemifields. The subjects' task was to detect in which hemifield the target was presented (twoalternative forced choice).

\section{Rhythmic visual stimulation results in hemifield-specific periodic modulation of perception}

The target stimulus could appear either at the side of the periodic stimulation (an "entrained" trial), or at side of the arhythmic stimulation (a "nonentrained" trial). Our main question was whether the delay between the last flash of the stimulation train and the target modulated perception rhythmically. First, we observed similar hit rates and reaction times for the entrained and nonentrained trials across subjects [hit rates: entrained trials, $80.5 \pm 1.0 \%$; nonentrained trials, $80.0 \pm 0.9 \%$ (mean $\pm \mathrm{SEM}$ ); paired-samples $t_{18}=0.54, p=0.60$; reaction times: entrained trials, $362 \pm 55 \mathrm{~ms}$; nonentrained trials, $356 \pm 55 \mathrm{~ms} ; t_{18}=0.96$, $p=0.35$ ], indicating that there was no difference in attention to an entrained versus a nonentrained hemifield. Hit rates strongly increased with longer delays (Fig. $1 B$ ), both for the entrained and nonentrained trials (Pearson correlation between delay and hit rate: entrained, $r=0.84$; nonentrained, $r=0.89$, both $p<10^{-5}$ ). This is expected because of the forward masking effect of the stimulus trains.

The hit rate difference between entrained trials and nonentrained trials revealed a clearly periodic pattern (Fig. 1C), indicating that the periodic stimulation resulted in a rhythmic modulation of detection performance matching the stimulation in frequency. We tested this periodicity by fitting a $10 \mathrm{~Hz}$ sinusoid to the hit rate data (Fig. $1 C$, green curve). The sinusoid plus a linear trend fitted the data significantly better than a linear trend only. This was assessed using a Wald $F$ test $\left(F_{(2,15)}=6.73, p=\right.$ $0.008)$, which controls for the increase in degrees of freedom. To alleviate possible concerns regarding a bias of the Wald test, we verified this observation by performing a permutation test, obtaining similar results $(p=0.01)$. Finally, to rule out the possibility that this result was driven by a small number of subjects, we performed a bootstrap procedure, obtaining a 95\% confidence interval on the circular-to-linear correlation between delay (expressed as $10 \mathrm{~Hz}$ phase) and hit rate of $r=0.46-0.91$, thus confirming a robust periodicity in the hit rate profile. Interestingly, we observed this periodicity after the offset of the rhythmic stimulation, indicating that the rhythmicity is maintained by an endogenous mechanism. Also note that the hit rate profile went both above and below the zero line (Fig. 1C). This means that perception was impaired at certain phases relative to the entraining stimuli, while it was enhanced at others.

Because the above analysis was based on the difference between the entrained and nonentrained trials, we cannot yet be certain that the periodicity in hit rate is exclusively explained by the rhythmic entrainment. To address this issue, we conducted a 
$2 \times 2$ repeated-measures ANOVA, with entrained versus nonentrained and inphase versus anti-phase as factors (Fig. $1 D)$. In-phase trials are here defined as those trials in which the target appeared precisely 100 or $200 \mathrm{~ms}$ after the entrainment; while anti-phase trials are those with a delay of 150 or $250 \mathrm{~ms}$. We found a significant interaction between entrainment and phase $\left(F_{(1,18)}=6.28, p=0.02\right)$. Post hoc $t$ tests revealed that there was a significant difference between in-phase and anti-phase hit rate for the entrained trials $\left(t_{18}=2.75, p=0.01\right)$, but this difference was not significantly present for the nonentrained trials $\left(t_{18}=0.65, p=\right.$ $0.53)$. These results demonstrate that the periodic modulation of detection performance is present only in the visual hemifield that received rhythmic visual stimulation.

\section{Rhythmic visual stimulation causes persistent alpha activity in early visual cortex}

We next asked whether the rhythmic stimulation entrained the alpha activity as detected in the MEG signal. We first report the event-related fields (ERFs) timelocked to the onset of the stimulation trains (Fig. 2). All ERFs are presented as planar gradient (two planar gradient orientations were combined using a singular-value decomposition approach, which preserves phase information). The periodic stimulus trains were accompanied by clear rhythmic temporal dynamics in the ERFs (Fig. 2). As expected, the ERFs with the highest amplitude occurred in the sensors contralateral to the rhythmic stimulation (occipital/posterior channels, as defined by the CTF MEG manufacturer; mean rectified ERF be-

tween 1.55 and $2 \mathrm{~s}$, contralateral vs ipsilateral sensors: $t_{18}=3.39$, $p=0.003)$. Some rhythmic activity was also observed contralateral to the jittered stimulation trains. This effect is best explained by field spread from the other hemisphere (Lütkenhöner, 2003). Importantly, we found that the phase-locked rhythmic brain activity persisted after the offset of the rhythmic visual stimulation (Fig. 2, second vertical dotted line). These results do not change qualitatively when we compute the ERF only for trials where the target appeared $\geq 200 \mathrm{~ms}$ after the rhythmic stimuli offset (data not shown). We therefore conclude that the effects are not driven by target processing in the poststimulation interval. Statistical assessment of the phase-locking evident in the ERFs is more straightforward when based on the time-frequency representation of ITC; this is described below.

Next we identified the effects of the rhythmic stimulation in the frequency domain. The time-frequency representations of power averaged over trials showed a clear effect in the alpha band (Fig. 3A). Considering the power difference between sensors contralateral and ipsilateral to the entrained hemifield, we found that the alpha activity was strongest in the contralateral sensors lasted the rhythmic stimulation.

\section{Power}

Inter-trial coherence (ITC)
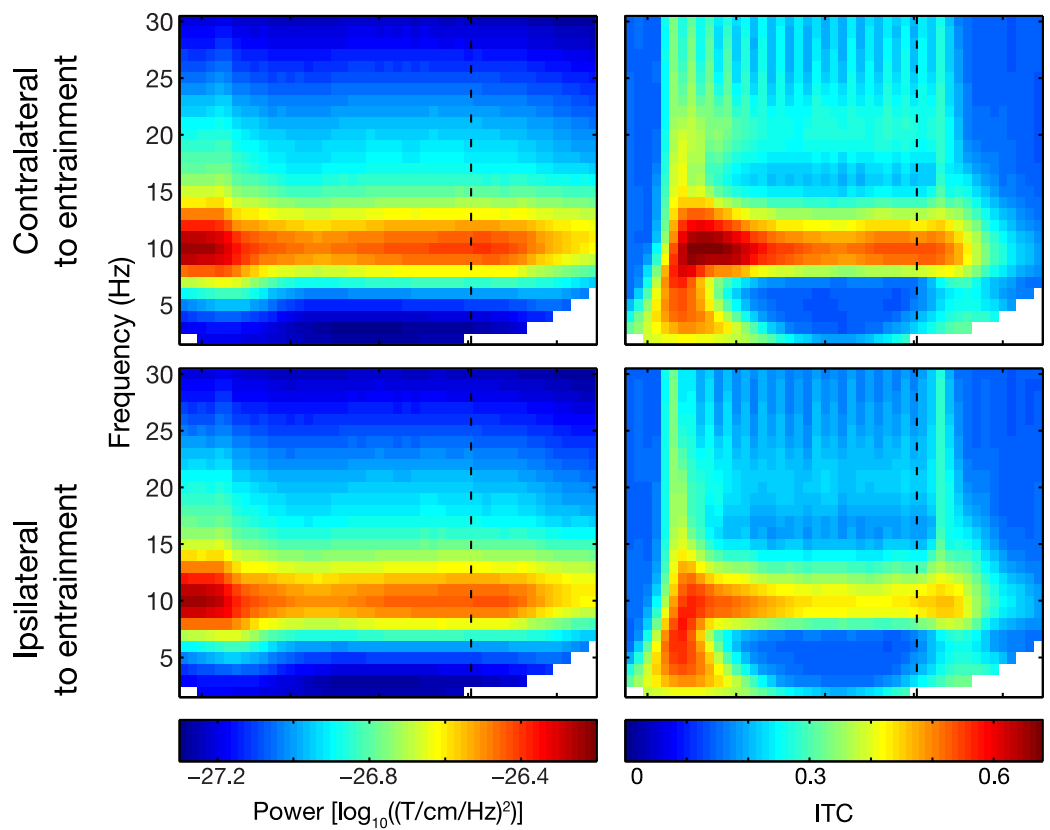

Time (s)

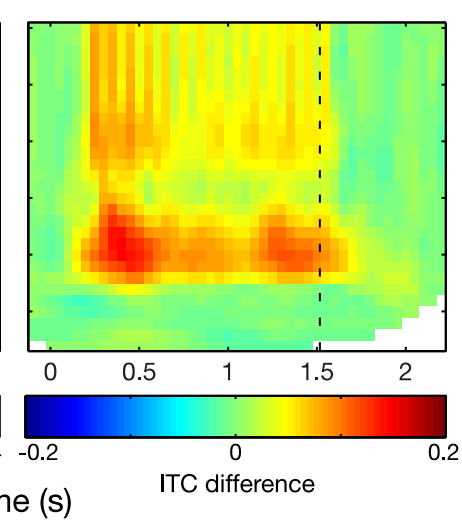

Time (s)

Figure 3. Frequency-domain analysis results. Time-frequency representations of power (left) and ITC (right), shown for sensors contralateral (top) and ipsilateral (middle) to the rhythmic stimulation. The bottom plots show the difference between contralateral and ipsilateral sensors. A persistent alpha power and ITC increase was visible for contralateral sensors, which out-

[cluster-based permutation test (Maris and Oostenveld, 2007), $p=0.004]$, confirming the entrainment of alpha activity. We also found a clear reduction in low-frequency activity in the contralateral sensors (cluster-based permutation test, $p=0.001$ ). The 10 $\mathrm{Hz}$ power in ipsilateral sensors is best explained by magnetic field spread of activity from the other hemisphere (as confirmed by source analysis below; Lütkenhöner, 2003). A similar pattern was observed when considering the ITC (Fig. $3 A$, right): alpha band ITC is significantly higher for the contralateral sensors $(p=$ 0.001). From Figure 3 (bottom left), it seems clear that the entrained activity persists after the stimulus train. To exclude effects from the temporal smoothing introduced by the spectral analysis, we also computed the alpha power and ITC for the MEG in the postentrainment interval only $(1.5-2 \mathrm{~s})$. We found a significantly greater $10 \mathrm{~Hz}$ power $\left(t_{18}=3.32, p=0.004\right)$ and ITC $\left(t_{18}=5.01\right.$, $\left.p=9.1 \cdot 10^{-5}\right)$ postentrainment for the sensors contralateral to the entrainment hemifield than for the ipsilateral sensors. Similar results are obtained when we test alpha power and ITC in a narrow time window starting $200 \mathrm{~ms}$ after the stimulation $(1.7-1.8 \mathrm{~s}$; power: $t_{18}=2.06, p=0.054$; ITC: $t_{18}=2.88, p=0.01$ ). Finally, 


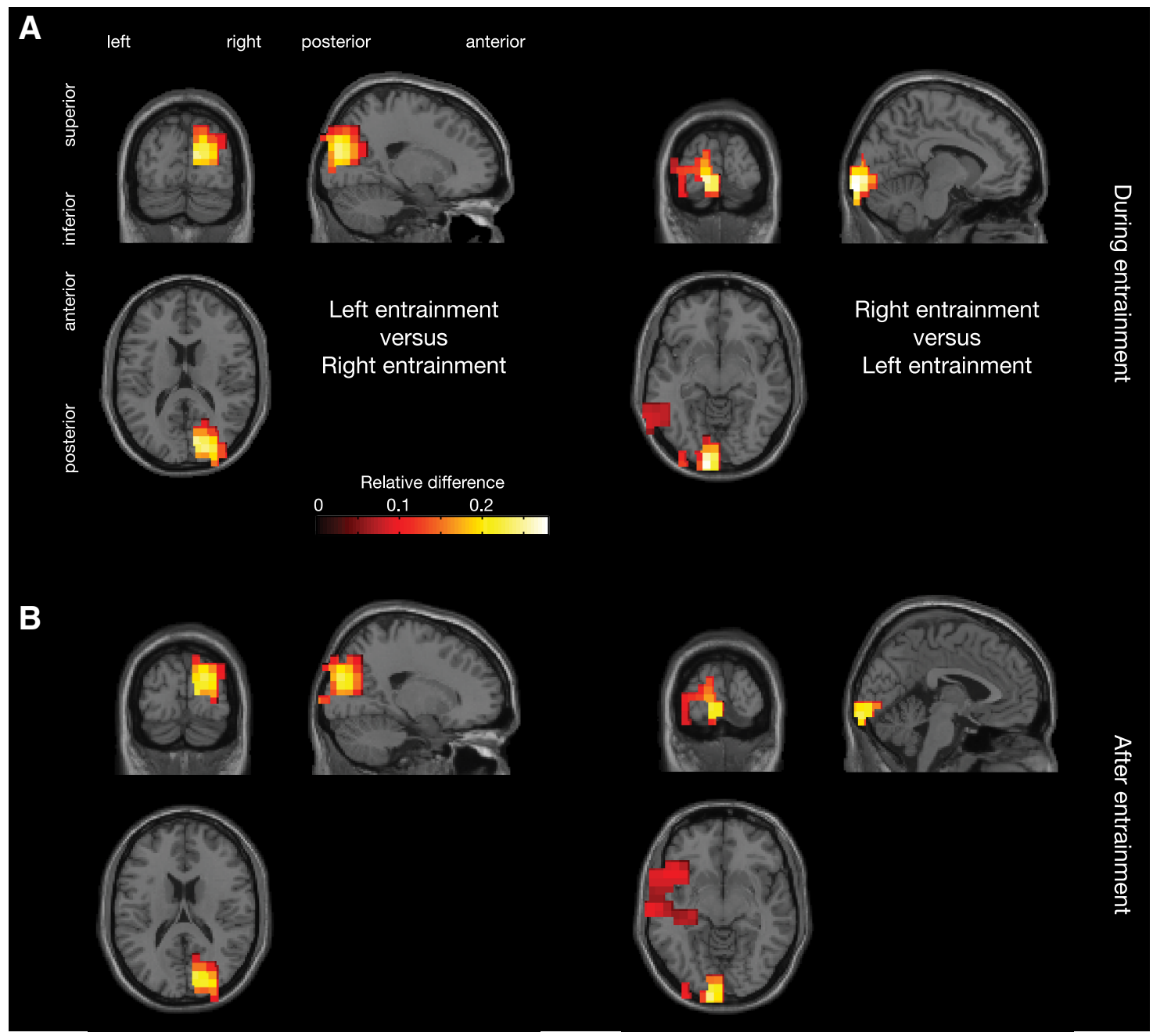

Figure 4. Source analysis results. $A$, Grand-average results for DICS beamformer source analysis of entrained $10 \mathrm{~Hz}$ activity during stimulation, interpolated onto a single-subject MNI template brain. Values are masked with cluster-based permutation test $p<0.05$, controlling for multiple comparisons. The left and right panels show the sources for the $10 \mathrm{~Hz}$ activity during left and right rhythmic stimulation, respectively. $\boldsymbol{B}$, Same, but with activity estimated during the postentrainment window.

we also calculated ITC from Hilbert-transformed, bandpassfiltered data. This analysis confirmed a sustained elevation of ITC after the stimulus train. The ITC increase was sustained for $\sim 200$ ms longer in the hemisphere contralateral to the entraining stimuli than in the ipsilateral hemisphere (data not shown). We conclude that the rhythmic stimulation resulted in increased endogenous phase-locked alpha activity persisting after the stimulation.

To identify the neural sources corresponding to the alpha entrainment, we performed a beamformer analysis (DICS; Gross et al., 2001) on the MEG data at $10 \mathrm{~Hz}$ in the interval 0.9-1.5 s. Figure $4 A$ shows the contrast between left and right entrainment in the left panel, and the reverse contrast in the right panel. We show only the significant clusters $(p<0.05)$ arising from a cluster-based permutation test (Maris and Oostenveld, 2007), controlling for multiple comparisons. For both the left and the right entrainment, there were clear peaks in the occipital cortices contralateral to the entrained hemifield, around the calcarine sulci. When we apply the spatial filter computed during the stimulation to the activity in the time window after stimulation, 1.5-2 $s$, the same neural sources are significantly activated (Fig. $4 B$; masked with cluster-permutation $p<0.05$ ). These results indicate that early visual regions are responsible for the alpha power increase observed both during and after the rhythmic $10 \mathrm{~Hz}$ stimulation.

\section{Periodic modulation of perception is mediated by entrained alpha activity}

We next asked whether variability in alpha entrainment over trials as observed in the recorded MEG signal predicted the periodicity found in the detection performance. We quantify this periodicity by an entrainment index (EI), which reflects the amount to which the rhythmic stimulus train caused a rhythmic effect in the hit rate (see Materials and Methods). We separated the trials for each subject into four bins corresponding to quartiles of alpha power during the periodic stimulus trains, and computed the EI separately for trials belonging to each of those bins (Fig. 5). The alpha power used to sort the trials was estimated either from the sensors contralateral to the entrainment stimuli (where, as shown, the strongest alpha effect was observed), or from the ipsilateral sensors. We found a significant interaction between the alpha-providing hemisphere and alpha quartile $\left(F_{(3,18)}=2.86, p=0.045\right)$. Subsequent $t$ tests revealed that higher alpha quartiles corresponded to a higher EI, but only when alpha power was estimated from the sensors contralateral to rhythmic stimulation (fourth quartile vs first quartile: $t_{18}=2.29, p=0.03$ ). 
Alpha power taken from contralateral sensors

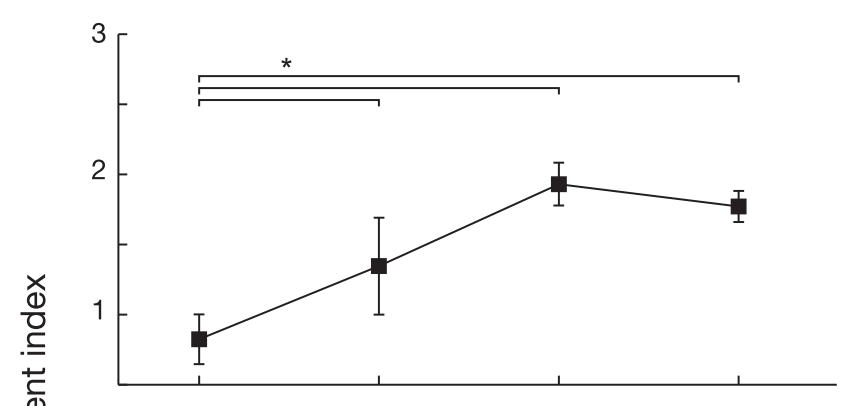

Alpha power taken from ipsilateral sensors

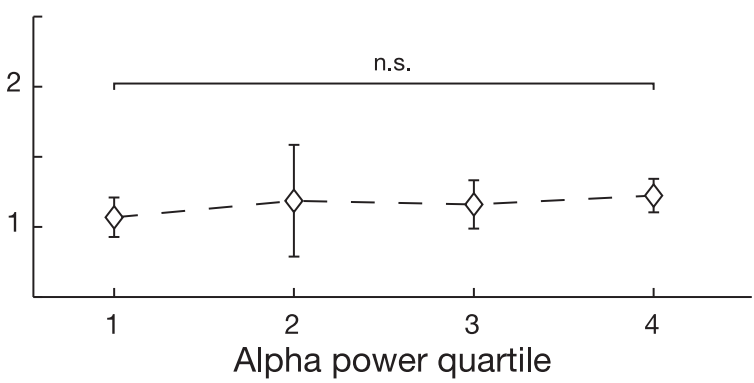

Figure 5. Relation between entrained alpha activity and perception. The entrainment index, i.e., the amount to which the rhythmic stimuli resulted in a rhythmic hit rate modulation, shown separately for four quartiles of alpha power. Alpha power was estimated either from the sensors ipsilateral (bottom) or contralateral (top) to the rhythmic stimulation. Importantly, the hit rate was strongly phasically modulated for trials during which contralateral entrained alpha power was high, but not for trials with low power. No such effect was observed for the ipsilateral alpha. Error bars reflect unbiased within-subjects corrected SEM (Cousineau, 2005; Morey, 2008). ${ }^{*} p<0.05$.

This was not the case when alpha power was estimated from the sensors ipsilateral to rhythmic stimulation (fourth quartile vs first quartile: $t_{18}=0.71, p=0.49$ ). We conclude that higher locally entrained alpha power predicts a stronger phasic effect in detection performance.

To rule out the possibility that this correlation between perceptual rhythmicity and alpha power entrainment is trivially explained by effects of vigilance (which could be reflected in a general alpha power increase or decrease), we also computed the ITC separately for the different alpha power quartiles. We find a significant effect of alpha power quartile on $\operatorname{ITC}\left(F_{(3,18)}=7.22\right.$, $p=4 \cdot 10^{-4}$, with higher alpha power quartiles corresponding to higher ITC (fourth quartile vs first quartile: $t_{18}=3.11, p=$ $0.006)$. We can therefore conclude that the rhythmic modulation of hit rate is due to entrainment of endogenous oscillations in the alpha band.

\section{Discussion}

We have demonstrated that $10 \mathrm{~Hz}$ rhythmic visual stimulation induces alpha-band rhythmicity in ongoing neuronal activity. The entrained rhythm was intrinsic to the brain, since it outlasted the stimulation train by several cycles. Furthermore, the stimulation caused a subsequent rhythmicity in subjects' perceptual ability. Importantly, the degree of rhythmic entrainment of the MEG signal predicted the rhythmicity in perceptual performance. The rhythmic modulation of both the brain activity and the detection performance was limited to the hemisphere contralateral to the stimulated hemifield, suggesting local neuronal entrainment. This was confirmed by source localization demonstrating the involvement of early visual cortical areas.
Previous work has convincingly demonstrated that trains of TMS pulses and visual stimuli can entrain brain activity detected in the EEG (Thut et al., 2012). It has been shown that $\sim 10 \mathrm{~Hz}$ visual stimulation results in the strongest neural entrainment compared with other frequency bands (Herrmann, 2001). Furthermore it has been shown that $10 \mathrm{~Hz}$ entrainment can produce a cyclic modulation of perceptual performance; however, the brain dynamics underlying this phenomenon are still unclear (Mathewson et al., 2012; de Graaf et al., 2013). We now directly link the measured entrainment to behavior: the entrained brain activity is correlated over trials with the rhythmicity in the periodic hit rate profile. Another important insight from our findings is that the entrainment is local; i.e., it reflects the rhythmic engagement of spatially specific visual regions rather than a more global signal. This claim is based on the fact that the effect on target perception was only seen in the rhythmically stimulated hemifield. The MEG data corroborated this finding: the sources reflecting the entrained activity were localized in the hemisphere contralateral to the rhythmic stimulation. This result is consistent with studies using TMS to entrain respectively the left or right hemispheres (Romei et al., 2010); we now provide evidence that local entrainment of brain activity in the visual cortex can be obtained using visual stimulus trains [similar results seem to hold for frequency modulation of sound entraining the auditory cortex (Henry and Obleser, 2012)]. An interesting hypothesis is that the alpha entrainment by visual stimulation we report here is responsible for the phenomenon known as the "attentional blink"; recently, evidence for this hypothesis has been reported (Zauner et al., 2012).

A recent study (Jaegle and Ro, 2014) also reported entrainment of alpha activity (as measured by EEG) using TMS to either occipital or parietal cortex. Interestingly, however, they report a behavioral effect of their entrainment only when TMS was applied to the parietal cortex. This is in line with another TMS study reporting alpha entrainment after parietal stimulation (Thut et al., 2011). We report strong, behaviorally relevant, occipital alpha entrainment. Although the studies cited above (and others, e.g., Hanslmayr et al., 2013) show convincing evidence for the existence of a parietal alpha source, there is ample evidence that early visual cortex also contains alpha generators (Lopes Da Silva and Storm Van Leeuwen, 1977; Bollimunta et al., 2008; Spaak et al., 2012) that are modulated with attention (Yamagishi et al., 2003; Bollimunta et al., 2011). It is conceivable that intrinsically occurring alpha modulations, such as those occurring in response to attentional cues, are top-down controlled by parietal cortex, while the consequences of this modulation are expressed in occipital areas. Given the medial and deep location of the primary visual cortex, it might not be easily entrainable by TMS, while the dorsal and lateral location of the parietal areas associated with alpha generation (surrounding the intraparietal sulcus) is anatomically ideal for transcranial stimulation. Entrainment using visual flashes as reported here is of course not subject to such anatomical constraints, as the stimulation is relayed directly by existing endogenous pathways.

The hit rate profile we observed was clearly periodic. It might be argued that these behavioral effects are not due to low-level entrainment of endogenous oscillators, but instead reflect temporal expectation. However, temporal expectation cannot explain our results, because it would predict the opposite pattern: subjects performed worse at in-phase delays (100/200/300 ms; Fig. $1 C, D)$ than at anti-phase delays. Since the entraining stimuli were presented at $0,-100,-200, \ldots \mathrm{ms}$, the temporal expectation hypothesis would predict an increased performance at in- 
phase delays (Rohenkohl and Nobre, 2011; Rohenkohl et al., 2012; Cravo et al., 2013). Presumably temporal expectation is associated with different neural mechanisms than those reflecting the entrainment we observed.

This brings us to an important point: it seems that (at least) two connotations of "entrainment" are used in the literature. Temporal predictions and expectations are typically associated with entrainment of low-frequency neural oscillations (Lakatos et al., 2008; Schroeder and Lakatos, 2009; Cravo et al., 2013). This sense of "entrainment" likely reflects an active mechanism by which the brain extracts temporal regularities from the environment. In contrast, the entrainment of alpha activity that the current paper (and the cited papers on visual and TMS entrainment) is concerned with, is believed not to reflect such an active mechanism. Instead, this form of entrainment is likely a lower-level process that taps into kinetics of the neural system not specifically evolved to extract temporal information; this latter form of entrainment is more akin to resonance of endogenous neural oscillators.

Is the entrainment specific to $\sim 10 \mathrm{~Hz}$ stimulation, or would stimulation at other frequencies have worked equally well? Importantly, since the entrained $10 \mathrm{~Hz}$ activity was sustained after the stimulus train for $\geq 300 \mathrm{~ms}$, it is clear that the induced rhythm is maintained by endogenous mechanisms. Given the large amplitude of spontaneous alpha oscillations, we argue that the sustained entrained oscillations rely on the same mechanisms as the natural alpha oscillators. In support of this notion, previous entrainment studies targeting the visual system have shown that perceptual entrainment is maximal at $\sim 10 \mathrm{~Hz}$ (Romei et al., 2010; de Graaf et al., 2013), and that even nonrhythmic input can result in cortical $10 \mathrm{~Hz}$ resonance (VanRullen and Macdonald, 2012). Also, rhythmic stimulation at frequencies other than 10 $\mathrm{Hz}$ was shown to induce a $10 \mathrm{~Hz}$ rhythmicity in perceptual performance (de Graaf et al., 2013, their Experiment 2). Furthermore, an exhaustive test of many different visual stimulation frequencies has revealed that $10 \mathrm{~Hz}$ activity in the visual system shows by far the strongest resonance (Herrmann, 2001). Finally, although it has convincingly been argued that steady-state responses during stimulation are largely explained by a superposition of transient responses (Capilla et al., 2011), we note that our arguments in favor of entrainment are based primarily on effects observed after stimulation offset, when the superposition of transients would have already disappeared. We conclude that the 10 $\mathrm{Hz}$ visual stimulation used in the present study most likely entrains the neuronal generators responsible for creating the spontaneous alpha activity.

Our results provide evidence for the hypothesis that perception unfolds not in a fully continuous manner, but is instead at least partly discrete (VanRullen and Koch, 2003; Schroeder and Lakatos, 2009; Schroeder et al., 2010). The discrete sampling of visual stimuli has been proposed to be clocked by an internal oscillatory rhythm (Busch et al., 2009; Landau and Fries, 2012; Romei et al., 2012; Fiebelkorn et al., 2013), and we here add evidence indicating that alpha oscillations are involved in this clocking of perception. Previous work has found that oscillatory EEG activity with a frontocentral topography is most predictive of perception (Busch et al., 2009), yet here we report occipital alpha to be the critical factor. The frontal versus posterior components might reflect complementary mechanisms of temporal sampling, or they might interact to result in the observed perceptual modulation. Alternatively, since the study by Busch et al. (2009) did not perform source analysis, it could also be the case that the observed EEG topography does not reflect the engage- ment of frontal sources. Further research is needed to shed light on the relation between a possible global rhythmicity in perception or attention, and the strongly localized oscillatory sampling we report here.

Most studies concerning the role of brain oscillations in perceptual and cognitive processes have relied on external cognitive cues to modulate the oscillations under scrutiny; e.g., a cue to covertly shift attention to one visual hemifield reliably induces a hemispheric lateralization in alpha band activity (Worden et al., 2000; Kelly et al., 2006; Rihs et al., 2007). Typical research questions have investigated how changes in neural oscillations affect cognitive performance. While these studies have provided important insights, claims made in this manner about the functional relevance of oscillations are correlative: the observed oscillations could be a consequence of some unobserved factor (which was the result of the cognitive cue), while this unobserved factor was also the cause of the cognitive change. A direct manipulation of endogenous brain oscillations allows researchers to make causal claims. We have here demonstrated that such a manipulation in the visual system is feasible by using visual stimulation.

Through using the technique of visual alpha entrainment, our findings show that cortically highly localized alpha activity modulates visual perception in a phase-specific manner. It has been demonstrated that alpha oscillations are under strong top-down control when attention is allocated (Haegens et al., 2011; Händel et al., 2011; Bonnefond and Jensen, 2012). They may therefore function as a mechanism for rhythmic gain control of neuronal processing (Arnal and Giraud, 2012; Jensen et al., 2012; Klimesch, 2012), possibly through interaction with gamma activity (Spaak et al., 2012; Lisman and Jensen, 2013). We show here that local alpha oscillations indeed are doing causal work; they are not an epiphenomenal consequence of some other attentional process. In future work, it would be of great interest to investigate how the allocation of attention interacts with the entrained alpha oscillations.

\section{References}

Arnal LH, Giraud AL (2012) Cortical oscillations and sensory predictions. Trends Cogn Sci 16:390-398. CrossRef Medline

Bastiaansen MC, Knösche TR (2000) Tangential derivative mapping of axial MEG applied to event-related desynchronization research. Clin Neurophysiol 111:1300-1305. CrossRef Medline

Bollimunta A, Chen Y, Schroeder CE, Ding M (2008) Neuronal mechanisms of cortical alpha oscillations in awake-behaving macaques. J Neurosci 28:9976-9988. CrossRef Medline

Bollimunta A, Mo J, Schroeder CE, Ding M (2011) Neuronal mechanisms and attentional modulation of corticothalamic alpha oscillations. J Neurosci 31:4935-4943. CrossRef Medline

Bonnefond M, Jensen O (2012) Alpha oscillations serve to protect working memory maintenance against anticipated distracters. Curr Biol 22:19691974. CrossRef Medline

Busch NA, Dubois J, VanRullen R (2009) The phase of ongoing EEG oscillations predicts visual perception. J Neurosci 29:7869-7876. CrossRef Medline

Capilla A, Pazo-Alvarez P, Darriba A, Campo P, Gross J (2011) Steady-state visual evoked potentials can be explained by temporal superposition of transient event-related responses. PLoS One 6:e14543. CrossRef Medline

Capotosto P, Babiloni C, Romani GL, Corbetta M (2009) Frontoparietal cortex controls spatial attention through modulation of anticipatory alpha rhythms. J Neurosci 29:5863-5872. CrossRef Medline

Capotosto P, Babiloni C, Romani GL, Corbetta M (2012) Differential contribution of right and left parietal cortex to the control of spatial attention: a simultaneous EEG-rTMS study. Cereb Cortex 22:446-454. CrossRef Medline

Cousineau D (2005) Confidence intervals in within-subjects designs: a simpler solution to Loftus and Masson's method. Tutor Quant Methods Psychol 1:42-45. 
Cravo AM, Rohenkohl G, Wyart V, Nobre AC (2013) Temporal expectation enhances contrast sensitivity by phase entrainment of low-frequency oscillations in visual cortex. J Neurosci 33:4002-4010. CrossRef Medline

de Graaf TA, Gross J, Paterson G, Rusch T, Sack AT, Thut G (2013) Alphaband rhythms in visual task performance: phase-locking by rhythmic sensory stimulation. PLoS One 8:e60035. CrossRef Medline

Fiebelkorn IC, Saalmann YB, Kastner S (2013) Rhythmic sampling within and between objects despite sustained attention at a cued location. Curr Biol 23:2553-2558. CrossRef Medline

Fox J (1997) Applied regression analysis, linear models, and related methods. Thousand Oaks, CA: SAGE.

Gross J, Kujala J, Hamalainen M, Timmermann L, Schnitzler A, Salmelin R (2001) Dynamic imaging of coherent sources: studying neural interactions in the human brain. Proc Natl Acad Sci U S A 98:694-699. CrossRef Medline

Haegens S, Händel BF, Jensen O (2011) Top-down controlled alpha band activity in somatosensory areas determines behavioral performance in a discrimination task. J Neurosci 31:5197-5204. CrossRef Medline

Halbleib A, Gratkowski M, Schwab K, Ligges C, Witte H, Haueisen J (2012) Topographic analysis of engagement and disengagement of neural oscillators in photic driving: a combined electroencephalogram/magnetoencephalogram study. J Clin Neurophysiol 29:33-41. CrossRef Medline

Händel BF, Haarmeier T, Jensen O (2011) Alpha oscillations correlate with the successful inhibition of unattended stimuli. J Cogn Neurosci 23: 2494-2502. CrossRef Medline

Hanslmayr S, Aslan A, Staudigl T, Klimesch W, Herrmann CS, Bäuml KH (2007) Prestimulus oscillations predict visual perception performance between and within subjects. Neuroimage 37:1465-1473. CrossRef Medline

Hanslmayr S, Volberg G, Wimber M, Dalal SS, Greenlee MW (2013) Prestimulus oscillatory phase at $7 \mathrm{~Hz}$ gates cortical information flow and visual perception. Curr Biol 23:2273-2278. CrossRef Medline

Hari R, Salmelin R (1997) Human cortical oscillations: a neuromagnetic view through the skull. Trends Neurosci 20:44-49. CrossRef Medline

Henry MJ, Obleser J (2012) Frequency modulation entrains slow neural oscillations and optimizes human listening behavior. Proc Natl Acad Sci U S A 109:20095-20100. CrossRef Medline

Herrmann CS (2001) Human EEG responses to 1-100 Hz flicker: resonance phenomena in visual cortex and their potential correlation to cognitive phenomena. Exp Brain Res 137:346-353. CrossRef Medline

Jaegle A, Ro T (2014) Direct control of visual perception with phase-specific modulation of posterior parietal cortex. J Cogn Neurosci 26:422-432. CrossRef Medline

Jensen O, Bonnefond M, VanRullen R (2012) An oscillatory mechanism for prioritizing salient unattended stimuli. Trends Cogn Sci 16:200-206. CrossRef Medline

Kelly SP, Lalor EC, Reilly RB, Foxe JJ (2006) Increases in alpha oscillatory power reflect an active retinotopic mechanism for distracter suppression during sustained visuospatial attention. J Neurophysiol 95:3844-3851. CrossRef Medline

Klimesch W (2012) Alpha-band oscillations, attention, and controlled access to stored information. Trends Cogn Sci 16:606-617. CrossRef Medline

Lakatos P, Karmos G, Mehta AD, Ulbert I, Schroeder CE (2008) Entrainment of neuronal oscillations as a mechanism of attentional selection. Science 320:110-113. CrossRef Medline

Landau AN, Fries P (2012) Attention samples stimuli rhythmically. Curr Biol 22:1000-1004. CrossRef Medline

Lisman JE, Jensen O (2013) The $\theta-\gamma$ neural code. Neuron 77:1002-1016. CrossRef Medline

Lopes Da Silva FH, Storm Van Leeuwen W (1977) The cortical source of the alpha rhythm. Neurosci Lett 6:237-241. CrossRef Medline

Lütkenhöner B (2003) Magnetoencephalography and its Achilles' heel. J Physiol Paris 97:641-658. CrossRef Medline

Maris E, Oostenveld R (2007) Nonparametric statistical testing of EEG- and MEG-data. J Neurosci Methods 164:177-190. CrossRef Medline
Mathewson KE, Gratton G, Fabiani M, Beck DM, Ro T (2009) To see or not to see: prestimulus $\alpha$ phase predicts visual awareness. J Neurosci 29:27252732. CrossRef Medline

Mathewson KE, Prudhomme C, Fabiani M, Beck DM, Lleras A, Gratton G (2012) Making waves in the stream of consciousness: entraining oscillations in EEG alpha and fluctuations in visual awareness with rhythmic visual stimulation. J Cogn Neurosci 24:2321-2333. CrossRef Medline

Morey RD (2008) Confidence intervals from normalized data: a correction to Cousineau (2005). Tutor Quant Methods Psychol 4:61-64.

Nolte G (2003) The magnetic lead field theorem in the quasi-static approximation and its use for magnetoencephalography forward calculation in realistic volume conductors. Phys Med Biol 48:3637-3652. CrossRef Medline

Oostenveld R, Fries P, Maris E, Schoffelen JM (2011) FieldTrip: open source software for advanced analysis of MEG, EEG, and invasive electrophysiological data. Comput Intell Neurosci 2011:156869. CrossRef Medline

Rihs TA, Michel CM, Thut G (2007) Mechanisms of selective inhibition in visual spatial attention are indexed by alpha-band EEG synchronization. Eur J Neurosci 25:603-610. CrossRef Medline

Rohenkohl G, Nobre AC (2011) $\alpha$ Oscillations related to anticipatory attention follow temporal expectations. J Neurosci 31:14076-14084. CrossRef Medline

Rohenkohl G, Cravo AM, Wyart V, Nobre AC (2012) Temporal expectation improves the quality of sensory information. J Neurosci 32:8424-8428. CrossRef Medline

Romei V, Gross J, Thut G (2010) On the role of prestimulus alpha rhythms over occipito-parietal areas in visual input regulation: correlation or causation? J Neurosci 30:8692-8697. CrossRef Medline

Romei V, Gross J, Thut G (2012) Sounds reset rhythms of visual cortex and corresponding human visual perception. Curr Biol 22:807-813. CrossRef Medline

Schroeder CE, Lakatos P (2009) Low-frequency neuronal oscillations as instruments of sensory selection. Trends Neurosci 32:9-18. CrossRef Medline

Schroeder CE, Wilson DA, Radman T, Scharfman H, Lakatos P (2010) Dynamics of active sensing and perceptual selection. Curr Opin Neurobiol 20:172-176. CrossRef Medline

Spaak E, Bonnefond M, Maier A, Leopold DA, Jensen O (2012) Layerspecific entrainment of gamma-band neural activity by the alpha rhythm in monkey visual cortex. Curr Biol 22:2313-2318. CrossRef Medline

Thut G, Veniero D, Romei V, Miniussi C, Schyns P, Gross J (2011) Rhythmic TMS causes local entrainment of natural oscillatory signatures. Curr Biol 21:1176-1185. CrossRef Medline

Thut G, Miniussi C, Gross J (2012) The functional importance of rhythmic activity in the brain. Curr Biol 22:R658-R663. CrossRef Medline

van Dijk H, Schoffelen JM, Oostenveld R, Jensen O (2008) Prestimulus oscillatory activity in the alpha band predicts visual discrimination ability. J Neurosci 28:1816-1823. CrossRef Medline

VanRullen R, Koch C (2003) Is perception discrete or continuous? Trends Cogn Sci 7:207-213. CrossRef Medline

VanRullen R, Macdonald JS (2012) Perceptual echoes at $10 \mathrm{~Hz}$ in the human brain. Curr Biol 22:995-999. CrossRef Medline

Watson AB, Pelli DG (1983) QUEST: a Bayesian adaptive psychometric method. Percept Psychophys 33:113-120. CrossRef Medline

Worden MS, Foxe JJ, Wang N, Simpson GV (2000) Anticipatory biasing of visuospatial attention indexed by retinotopically specific alpha-band electroencephalography increases over occipital cortex. J Neurosci 20:RC63. Medline

Yamagishi N, Callan DE, Goda N, Anderson SJ, Yoshida Y, Kawato M (2003) Attentional modulation of oscillatory activity in human visual cortex. Neuroimage 20:98-113. CrossRef Medline

Zauner A, Fellinger R, Gross J, Hanslmayr S, Shapiro K, Gruber W, Müller S, Klimesch W (2012) Alpha entrainment is responsible for the attentional blink phenomenon. Neuroimage 63:674-686. CrossRef Medline 